\title{
Phenotypic Characters of Staphylococcus Aureus Isolates from Clinical Samples in Aminu Kano Teaching Hospital, Kano, Nigeria
}

\author{
Abdulhadi Sale Kumurya*, Hamza Sule, Nasidi Abba Ahmed \\ Department of Medical Laboratory Science, Faculty of Allied Health Sciences, Bayero University, Kano, Nigeria
}

Email address:

askumurya.med@buk.edu.ng (A. S. Kumurya), sule.hamza@yahoo.com (H. Sule)

${ }^{*}$ Corresponding author

\section{To cite this article:}

Abdulhadi Sale Kumurya, Hamza Sule, Nasidi Abba Ahmed. Phenotypic Characters of Staphylococcus Aureus Isolates from Clinical Samples in Aminu Kano Teaching Hospital, Kano, Nigeria. American Journal of Internal Medicine. Vol. 5, No. 5, 2017, pp. 74-78. doi: 10.11648/j.ajim.20170505.12

Received: April 19, 2017; Accepted: May 26, 2017; Published: August 11, 2017

\begin{abstract}
Bacterial colonies can differ greatly in their morphologies. These differences can help us in identifying different species of bacteria. The clinical isolates of Staphylococcus Aureus are subjected to standard biochemical tests, to observe the biochemical characteristics. One hundred and ten (110) pathogenic Staphylococcus Aureus strains were used in this study. Characteristics of these strains were determined by biochemical tests such as catalase, coagulase, DNase test, test for beta haemolysin, fermentation of mannitol and lactose. Staphylococcus Aureus [American Type Culture Collection (ATCC) 25923] was used as a reference control organism. From this study, males were more infected than females, having $60(61.8 \%)$ and 42 (38.2\%) respectively. The age group with the highest number of isolates was $(0-10)$ years while blood culture had the highest frequency of Staphylococcus Aureus isolates with a frequency of 42 (38.1\%). It was also observed that $100 \%$ of Staphylococcus Aureus showed positive results in catalase and tube coagulase, about $101(91.8 \%)$ were positive for slide coagulase production test, $102(92.7 \%)$ strains were positive to DNase test, $63(57.3 \%)$ were positive to beta haemolysin while 79 (71.8\%) and $106(96.4 \%)$ strains fermented Mannitol and lactose respectively. This study reveals that no single test that can be used for the identification of Staphylococcus Aureus, hence the need for sequel testing using proper biochemical tests should be used for the identification of Staphylococcus Aureus isolates.
\end{abstract}

Keywords: Staphylococcus Aureus, Biochemical, Characteristics, Fermentation, Identification

\section{Introduction}

Staphylococcus Aureus is a Gram-positive bacterium belonging to the family Staphylococcaceae and is often found as a commensal on the skin, skin glands and mucous membranes particularly in the nose of healthy individuals [20]. Staphylococcus Aureus has emerged as one of the most important human pathogens, being over the past several decades, a leading cause of hospital and community acquired infections [14]. It is associated with variety of clinical infections including septicemia, pneumonia, wound sepsis, septic arthritis, osteomyelitis and post-surgical toxic shock syndrome with substantial rates of morbidity and mortality $[4,5,21]$. It can cause infections commonly in newborns, surgical, burns, diabetic patients and persons who are taking drugs suppressing the immunodeficiency diseases [25].

In developing countries, phenotypic tests are routinely used in the diagnosis of staphylococcal infections, in which coagulase tests are usually confirmatory for Staphylococcus Aureus $[3,15]$. Several studies in Nigeria indicated that Staphylococcus Aureus is among the most frequently isolated bacteria in Nigeria [1, 19, 24].

Staphylococcus Aureus is usually isolated on non-specific media (e.g. nutrient agar) and then presumptively identified before definitive overnight characterization [12]. In an attempt to achieve presumptive isolation in a single step, mannitol salt agar (MSA) was developed in 1945 for the 
selective isolation of pathogenic staphylococci in the clinical microbiology laboratory. The growth and production of yellow colonies, due to the high salt content of the medium and fermentation of mannitol, is regarded as a presumptive tool in the identification of Staphylococcus Aureus. It is also described as a characteristic for the differentiation of coagulase-positive staphylococci from coagulase-negative staphylococci [7].

However, there are reports that some coagulase-negative staphylococci can also produce yellow colonies on mannitol salt agar [11, 22, 26].

Single phenotypic test is inefficient for the identification of Staphylococcus Aureus. However a combination of mannitol salt agar and DNAse improves the tube coagulase test [16].

Since Staphylococcus Aureus appears to be the major cause of both hospital and community acquired infections, it is important that proper identification and characterization of this organism is carried out. It was because of this that this study was carried out to characterize Staphylococcus Aureus isolated from clinical samples in Aminu Kano Teaching Hospital, Kano, Nigeria.

\section{Materials and Methods}

\subsection{Study Area}

This study was carried out in Medical Microbiology Laboratory of Aminu Kano Teaching Hospital and was restricted to the Staphylococcus Aureus Isolates obtained from various clinical samples processed in the laboratory.

\subsection{Sample Size}

The prevalence of Staphylococcus Aureus in clinical isolate was found to be $7 \%$ in a study of conventional and rapid methods for identification of Staphylococcus Aureus from clinical specimens at Zaria, Nigeria [18]. Thus for this study the prevalence was used to calculate sample size as follows

$$
\mathrm{n}=\frac{Z^{2} P q}{d^{2}}
$$

Where

$\mathrm{n}=$ number of samples

$Z=$ statistic for level of confidence at $95 \%=1.96$

$\mathrm{P}=$ prevalence $=7 \%(0.07)$

$\mathrm{D}=$ allowable error of $5 \%,(0.05)$

$\mathrm{q}=1-\mathrm{p}$

$$
\mathrm{n}=\frac{1.96^{2} \times 0.07(1-0.07)}{0.05^{2}}=100.03 \text { hence } \mathrm{n} \approx 110
$$

\subsection{Bacterial Isolates}

A total of 110 consecutive non-duplicated Staphylococcus Aureus isolates were obtained from various clinical samples such as wound swab, blood culture, eye swab, ear swab, throat swab, catheter tips and vaginal swab samples and was identified using standard bacteriological procedures [6]. The quality control and rejection criteria of specimen [10] were followed. Staphylococcus Aureus (ATCC 25923) was used as control in every test run.

\subsection{Identification of Isolates by Standard Bacteriological Procedures}

\subsubsection{Gram Stain}

Gram staining was carried out on all the bacterial isolates. A drop of sterile normal saline was placed on a well labeled clean grease-free glass slide using a sterile inoculating loop; a colony of an overnight culture of the bacterial isolate was emulsified with the sterile normal saline to make a thin smear. The smear was air dried and then heat fixed. The slide was flooded with crystal violet (primary stain) for $60 \mathrm{~s}$ after which the stain was rinsed from the slide with water. The smear was flooded with Lugol's iodine (mordant) to fix the primary stain. The smear was be rinsed with water after $30 \mathrm{~s}$. The slide was then flooded with acetone and rinsed off almost immediately. The counter stain; neutral red was added and left for $60 \mathrm{~s}$ before being rinsed off. The stained smear was air dried and then observed under the microscope using $\mathrm{X} 100$ oil immersion objective lens of the microscope. A cluster of purple round colonies is indicative of Staphylococci [6].

\subsubsection{Catalase Test}

Staphylococci produce catalase an important virulence factor which degrades the microbicidal (hydrogen peroxide) $\mathrm{H}_{2} \mathrm{O}_{2}$ into oxygen $\left(\mathrm{O}_{2}\right)$ and water $\left(\mathrm{H}_{2} \mathrm{O}\right)$. This ability differentiates Staphylococci from Streptococci.

A drop of $3 \%$ hydrogen peroxide was placed on a clean grease-free glass slide. About 2 colonies of the bacteria was picked from a culture plate using a sterile wire loop and placed on the hydrogen peroxide; presence of bubbles indicates a positive catalase test [6].

\subsubsection{Coagulase Test}

Staphylococcus Aureus is distinguished from other Staphylococci by the production of coagulase an enzyme that clots plasma [6].

\section{(i). Bound Coagulase}

About one or two drops of blood plasma was placed on a clean grease-free glass slide and 2 colonies of the organism were picked using a sterile wire loop from a culture plate. The colonies will be emulsified in the plasma and observation of a clot indicates a positive coagulase test [6].

\section{(ii). Free Coagulase}

Three small test tubes were labeled: ' $\mathrm{T}$ ' for Test organism (18h broth culture), 'P' for Positive control (18h Staphylococcus Aureus broth culture) and ' $\mathrm{N}$ ' for Negative control (sterile broth). About $0.2 \mathrm{ml}$ of serum was added into each tube, $0.8 \mathrm{ml}$ of the test organism broth culture will be added to tube labeled T, $\mathrm{P}$ and $\mathrm{N}$ each. The tubes was mixed gently, and incubated at $37^{\circ} \mathrm{C}$. After 2 hours, the tubes were examined for clotting [6]. 


\subsubsection{Sugar Fermentation Test}

\section{(i). Mannitol Salt Agar (MSA)}

Using a sterile wire loop a small colony of the test organism was inoculated onto the surface of Mannitol Salt Agar plate and streaked. The culture plate was incubated aerobically at $37^{\circ} \mathrm{C}$. The plate was examined for Mannitol fermentation after 24 hours [6].

\section{(ii). MacConkey Agar}

Using a sterile wire loop a small colony of the test organism was inoculated onto the surface of MacConkey Agar plate and streaked. The culture plate was incubated aerobically at $37^{\circ} \mathrm{C}$. The plate was examined for Lactose fermentation after 24 hours [6].

\subsubsection{Deoxyribonucleic Test (DNase Test)}

DNase plates was divided into 6 sections by drawing lines on its bottom and Using a sterile wire loop the media was spot inoculated on to a small area in the middle of the marked sections with both the Test and Control organisms. It was incubated at $37^{\circ} \mathrm{C}$ overnight. After 24 hours, the surface of the plate was flooded with weak $\mathrm{HCl}$ acid solution. The excess acid tipped off and then after 5 minutes the colonies was examined [6].

\subsubsection{Haemolysin Activity Test}

The test organism was inoculated on blood agar by an agar overlay method and incubated at $37^{\circ} \mathrm{C}$ for 24 hours. After 24 hours, the plates will be observed for the presence of haemolysis [6].

\section{Results}

Of the One hundred and ten isolates tested, 68 were from male patients $(61.8 \%)$ and 42 from female patients $(38.2 \%)$ (Table 1). The age group $0-10$ years has the highest percentage of the isolates (44\%). This is followed by $61-70$, $21-30,11-20$ and $51-60$ years with $27.2 \%, 20 \%, 5.5 \%$ and $1.8 \%$ respectively. The least was found in the age group of $31-40$ and $41-50$ years with both having $0.9 \%$ each (Table 2).

The source of isolates with the highest frequency was blood culture $(38.1 \%)$, followed by wound swab and eye swab with $32.7 \%$ and $8.2 \%$ respectively. Catheter tip, ear swab and throat swab have $4.6 \%$ each. The source with the least number of isolates were high vaginal swab and semen with both having $3.6 \%$ each (Table 3 ).

Of the One hundred and ten isolates tested, $57.3 \%$ showed beta hemolysis, $92.7 \%$ were DNase positive, $71.8 \%$ were Mannitol positive, $96.4 \%$ were lactose positive and all the isolates were positive for catalase. While only $91.8 \%$ were positive for the slide coagulase production test, $100 \%$ were positive for the tube coagulase test (Table 4).

Table 5 depicts the division of the 110 isolates into biotypes based on their ability to ferment Mannitol and lactose. Of the 110 isolates, biotypes I represent the 78 $(70.9 \%)$ strains that are able to ferment both sugars, biotypes II represent the $1(0.9 \%)$ that only fermented Mannitol but not lactose. Biotypes III represent those $27(24.5 \%)$ that fermented lactose but not Mannitol. Biotypes IV represent those $4(3.6 \%)$ that neither fermented Mannitol nor lactose.

Table 1. Showing distribution of Staphylococcus Aureus Isolates according to gender $(N=110)$.

\begin{tabular}{lll}
\hline GENDER & FREQUENCY & PERCENTAGE (\%) \\
\hline MALE & 68 & 61.8 \\
FEMALE & 42 & 38.2 \\
Total & 110 & 100 \\
\hline
\end{tabular}

Table 2. Showing distribution of Staphylococcus Aureus Isolates according to age group.

\begin{tabular}{lll}
\hline AGE GROUP (YEARS) & FREQUENCY & PERCENTAGE (\%) \\
\hline $0-10$ & 48 & 44 \\
$11-20$ & 6 & 5.5 \\
$21-30$ & 22 & 20 \\
$31-40$ & 1 & 0.9 \\
$41-50$ & 1 & 0.9 \\
$51-60$ & 2 & 1.8 \\
$61-70$ & 30 & 27.2 \\
Total & 110 & 100 \\
\hline
\end{tabular}

Table 3. Showing distribution of Staphylococcus Aureus according to source of isolates.

\begin{tabular}{lll}
\hline SOURCE & FREQUENCY & PERCENTAGE (\%) \\
\hline Blood cultures & 42 & 38.1 \\
Wound swab & 36 & 32.7 \\
Eye swab & 9 & 8.2 \\
Catheter tips & 5 & 4.6 \\
Ear swab & 5 & 4.6 \\
Throat swab & 4.6 & 5 \\
High vaginal swab & 4 & 3.6 \\
Semen & 4 & 3.6 \\
Total & 110 & 100 \\
\hline
\end{tabular}

Table 4. Showing biochemical and some carbohydrate fermentation characteristics of the Staphylococcus Aureus isolates.

\begin{tabular}{lll}
\hline Tests & No. positive & \% positive \\
\hline Catalase & 110 & 100 \\
Slide Coagulase & 101 & 91.8 \\
Tube Coagulase & 110 & 100 \\
DNase & 102 & 92.7 \\
Beta hemolysin & 63 & 57.3 \\
Mannitol & 79 & 71.8 \\
Lactose & 106 & 96.4 \\
\hline
\end{tabular}

Table 5. Showing distribution of Staphylococcus Aureus isolates into biotypes based on their ability to ferment mannitol and lactose.

\begin{tabular}{llll}
\hline Biotypes & Mannitol & Lactose & No. of positive (\%) \\
\hline I & + & + & $78(70.9)$ \\
II & + & - & $1(0.9)$ \\
III & - & + & $27(24.5)$ \\
IV & - & - & $4(3.6)$ \\
\hline
\end{tabular}

\section{Discussion}

The advance and increase of bacterial strains that are resistant to antibacterial drugs has emerged as a global 
problem [23]. In this study, males $(61.8 \%)$ were more infected than females $(38.2 \%)$, the reason for this is not clearly understood but this agrees with previous studies conducted at Aminu Kano Teaching Hospital [8].

Also in this study the highest frequency of isolates of Staphylococcus Aureus (44\%) was observed in the (0-10) year age group in which neonates and infants were included, concurring with previous studies conducted at Aminu Kano Teaching Hospital [8]. It is believed that their immunity is not properly developed at this stage to cope with bacterial infections hence they are vulnerable and easily infected especially when hospitalized. A study in Ilorin reported wound infections of $38 \%$ as the highest frequency of Staphylococcus Aureus isolates [17]. This is in contrast to the present study where the highest frequency was reported in blood culture $(38.1 \%)$, followed by wound swab $(32 \%)$.

In this study, $96.4 \%$ of the isolates showed a positive result in lactose fermentation test. Lactose fermenting bacteria usually developed into pink colours on the MacConkey agar plates because it contains crystal violet and bile salt [9]. This result is in slight agreement with previous studies conducted in 8 health institutions in 6 states in Northwestern Nigeria [13] where $100 \%$ of the isolates showed positive results in lactose fermentation. It was observed in this study that $71.8 \%$ fermented mannitol, $92.7 \%$ produced DNase and that $57.3 \%$ was positive to Beta hemolysin. This is in contrast to previous studies conducted in Zaria [18].

\section{Conclusion}

This study reveals that no single test that can be used for the identification of Staphylococcus Aureus, hence the need for sequel testing using proper biochemical tests should be used for the identification of Staphylococcus Aureus isolates. Staphylococcus Aureus appears to be the major cause of both hospital and community acquired infections, therefore it is important that proper identification and characterization of this organism is carried out.

\section{References}

[1] Ako-Nai, A. K, Ogunniyi, A. D., Lamikanra, A. B. and Torimiro, S. A. (1991). The Characterization of cilinical isolates of Staphylococcus Aureus in lie-Ife, Nigeria. Journal of Medical microbiology 32: 109-112.

[2] Ako-Nai, A. K., Lamikanra, A. B. and Onipede, A. O. (1995). Incidence of pathogenic Microorganism in clinical specimens from hospital in South-western Nigeria. East African Medical Journal 72(7): 436-441.

[3] Bello, C. S. S. and Qahtani, A. (2006). Pitfalls in the routine diagnosis of Staphylococcus Aureus. African Journal of Biotechnolgy. 4(1): 83-86.

[4] Boyce, J. M. (1997). Epidemiology and prevention of nosocomial infections. In: The Staphylococci in human disease. Edited by Crosslay KB and Archer AL Churchill Livingston, New York. 309-329.
[5] Casgrove, S. E., Sakoulas, G., Pemcevich E. N., Schwabar, M. J., Karchmer, A. W. and Carmeli. Y. (2003). Comparison of mortality associated with methicillin resistant and methicillin sensitive Staphylococcus Aureus bacteriemia: a meta analysis. Clinical Infectious Diseases. 36: 53-59.

[6] Cheesbrough M (2010). District Laboratory Practice in Tropical Countries part two, New York, Cambridge University Press U.S. pp. 64-67.

[7] Duguid, J. P. (1989). Cluster-forming Gram-positive cocci. In: Mackie and McCartney Practical Medical Microbiology, $13^{\text {th }}$ edn, Edited by J. G. Colle.

[8] Emmanuel, O. N. and Magaji, S. N. (2011). Antibiotic susceptibility pattern of Staphylococcus Aureus from Clinical Isolates in tertiary insititution in northern Nigeria. Pan African Medical Journal. 8: 4.

[9] Hass, D and Defago, G. (2005). Biological control of soilborne pathogens by fluorescent pseudomonads. Nat Rev Microbial. 3(4): 369-372.

[10] Isenberg, H. D. (1998). Agar screening test to detect oxacillin (methicillin) resistant Staphylococcus Spp. In: Essential procedures for clinical microbiology. 4: 232-234.

[11] Jayaratne, P. and Rutherford, C. (1999). Detection of methicillin resistant (MRSA) from growth on mannitol salt oxacillin agar using PCR for nosocomial surveillance. Diagnostic Microbioogy and Infectious Diseases. 35: 13-18.

[12] Kloos, W. E. and Bannerman, T. L. (1994). Update on clinical significance of coagulase-negative Staphylococci. Clinical Microbiology Reviews. 7: 117-140.

[13] Kumurya, A. S. (2015). Characters and Antibiotic Susceptibility Pattern of Typical and Atypical Staphylococcus Aureus Isolates from Clinical Samples in Northwestern Nigeria. Acta Velit. 1(3): 42-49. Available at www.actavelit.com.

[14] Lowy, F. D. (2003). Antimicrobial resistance: the example of Staphylococcus Aureus. Journal of Clinical Investigations. 111: $1265-1273$.

[15] Mugalu, J. (2006). "Aetiology, risk factors and immediate outcome of bacteriologically confirmed neonatal septicaemia in Mulago hospital, Uganda." African Health Sciences 6(2): 120-126.

[16] Najjuka, F. C., Kateete, D. P., Kimani, C. N., Katabazi, F. C., Okeng, A., Okee, M. S., Nanteza, A. and Joloba, M. L. (2010). Identification of: DNase and Mannitol salt agar improve the efficiency of the tube coagulase test. Annals of Clinical Microbiology and Antimicrobial, 9(23): 1186-1176.

[17] Ndip, R. N., Ebah, L. M. E. and Onile, B. A. 1997). Antibiogram of Staphylococcus Aureus from clinical Syndromes in Ilorin, Nigeria. Journal of Medical Laboratry Science. 6: 24-26.

[18] Nneoma, C. J. S. and Walter C. J. (2013). Conventional and Rapid Methods for Identification of Staphylococcus Aureus from Clinical Specimens. American Journal of Biomedical and Life Sciences. 1(3): 41-43.

[19] Oni, A. A., Bakare, R. A., Okesola, A. O., Ogunlowo, H. A. and Ewete, A. F. (1997). Partern of bacterial pathogens in surgical wounds infections. African Journal Medical Sciences. 26: 139-140. 
[20] Plata, K., Rosato, A. E. and Wegrzyn, G. (2009). Staphylococcus Aureus as an infectious agent: overview of biochemistry and molecular genetics of its pathogenicity. Acta Biochimica Polonica. 56: 597-612.

[21] Shopsin, B. and Kreiswirth, B. N. (2003). Molecular epidemiology of Methicillin-resistant Staphylococcus Aureus. Emerging Infections Disease 7: 323-326.

[22] Simor, A., Goodfellow, J., Loiue, L. and Loiue, M. (2001). Evaluation of a new medium, oxacillin resistance screening agar base, for the detection of methicillin-resistant from clinical specimens. Journal of Clinical Microbiology. 33: 3422 .
[23] Subhankari, P. C., Santanu, K. M., Somenath, R. (2011). Asian Pacific Journal of Tropical Medicine. 1(3): 212-216.

[24] Unachukwu, C. N., Obunge, O. K. and Odia, O. J. (2005). The bacteriology of diabetic foot ulcer in Port Harcourt, Nigeria. Nigerian Journal of Medicine. 14(2): 173-176./.

[25] Vlok, W. A. (1982). Essentials of medical microbiology. 2nd. J. B. Lippincott Philadelphia. 348-355.

[26] Zadik, P. M., Davies, S., Whittaker, S. and Mason, C. (2001). Evaluation of a new selective medium for methicillin resistant Staphylococcus Aureus. Journal of Medical Microbiology. 50: 476-479. 